\title{
Crimes Ambientais e Agentes Privados: Evidências para os Estados Brasileiros
}

\section{Environmental Crimes and Private Agents: Evidences for Brazilian States}

\author{
Júlia Gallego Ziero Uhr* \\ Daniel de Abreu Pereira Uhr**
}

Resumo: O objetivo deste artigo é apresentar evidências do papel dos agentes privados (ONGs ambientais e comunidades) no combate aos crimes ambientais no Brasil. Apresenta-se o modelo teórico de crime proposto por Gary Stanley Becker em sua obra Crime and punishment: an economic approach (1968), considerando o caso do meio ambiente. No modelo empírico, testa-se se os agentes privados impactam negativamente na oferta de crimes ambientais. Quanto à metodologia empírica, utiliza-se dados em painel para infrações ambientais registradas pelo Ibama nos estados brasileiros para os anos de 2006 e 2007. Os resultados empíricos confirmam que os agentes privados ajudam a mitigar os crimes ambientais no Brasil.

Palavras-chave: Teoria do crime. Meio ambiente. Dados em painel.

Abstract: The aim of this paper is to present evidences for the role of private agents (environmental NGOs and Communities) to combat crimes against the environment in Brazil. Gary Becker's theoretical crime model, as in Crime and Punishment: an economic approach, published in 1968, is presented considering the environmental case. In the empirical model it is tested whether private stakeholders negatively impact the environmental crime supply. As for the empirical methodology, panel data is used for environmental violations registered by Ibama in the Brazilian states for the years 2006 and 2007. The empirical results confirm that private agents help to mitigate the environmental crimes in Brazil.

Keywords: Crime theory. Environment. Panel data.

JEL Classification: D60; K42; Q50.

Doutora em Economia pela Universidade de Brasília (UnB). Professora do Departamento de Economia e do Mestrado em Economia Aplicada da Universidade Federal de Pelotas (UFPel). E-mail: julia.uhr@ufpel.edu.br

** Doutor em Economia pela Universidade de Brasília (UnB). Professor do Departamento de Economia e do Mestrado em Economia Aplicada da Universidade Federal de Pelotas (UFPel). E-mail: daniel. uhr@ufpel.edu.br 


\section{1 lntrodução}

Os trabalhos que estudam o comportamento dos agentes criminosos normalmente utilizam a análise teórica de Becker (1968) como ponto de partida, na qual o crime é entendido como uma atividade econômica e, portanto, é baseado na racionalidade do agente. A conclusão básica desse modelo é que o criminoso responde tanto à probabilidade de condenação quanto à severidade da punição se pego e condenado. Neste trabalho, o modelo de Becker (1968) é utilizado para analisar a oferta de crimes ambientais e como esta é influenciada pela ação privada.

Diversos trabalhos ressaltam a importância dos agentes privados no combate ao crime ambiental. Alguns dos agentes privados citados pela literatura são as organizações não governamentais ambientais (ONGs ambientais) e as próprias comunidades locais (DASGUPTA et al., 2000; AFSAH et al., 1996; FERRAZ; SEROA DA MOTTA, 2002). Desse modo, utilizam-se tanto as ONGs ambientais quanto as comunidades como proxies para os agentes privados. $\mathrm{O}$ argumento para o uso dessas variáveis é que a ação privada pode aumentar a probabilidade de condenação de um criminoso, através do monitoramento, além de atuar diretamente sobre as sanções (punições informais), tais como estigma moral e perdas de reputação (COHEN, 2000; ALMER; GOESCHL, 2010).

O objetivo deste artigo é apresentar evidências teóricas e empíricas para o papel dos agentes privados no combate a crimes contra o meio ambiente. Na análise teórica, descreve-se o problema do crime ambiental através do arcabouço teórico proposto por Becker (1968), em especial ressaltando como a oferta de crimes ambientais é afetada pelos agentes privados. Na análise empírica, a hipótese de que os agentes privados impactam negativamente a oferta de crimes ambientais é testada para o Brasil. Para tanto, utilizam-se métodos de dados em painel para infrações ambientais registradas pelo Instituto Brasileiro do Meio Ambiente e dos Recursos Naturais Renováveis (Ibama) para os estados brasileiros nos anos de $2006 \mathrm{e}$ 2007. A variável "ONGs" é dada pelo número de ONGs ambientais com sede em cada estado cadastradas junto ao Ministério do Meio Ambiente no respectivo ano, e a variável "comunidade" ${ }^{1}$ caracteriza a concentração populacional do estado em centros urbanos, ou seja, é formada pela razão entre população urbana e o total da população estadual no respectivo ano. O resultado empírico corrobora o argumento de que os agentes privados ajudam a mitigar a oferta de crimes ambientais. Assim, este trabalho inova na medida em que proporciona evidências da importância dos agentes privados no combate ao crime ambiental no Brasil.

$\overline{1}$ Medida através da taxa de urbanização do estado. A hipótese é que, quanto maior a taxa de urbanização, mais fácil é o monitoramento de firmas poluidoras através da troca de informações entre os indivíduos da sociedade. 
Este artigo está estruturado da seguinte maneira: a seção 2 apresenta o modelo de teoria econômica do crime proposto por Becker (1968), aplicado para as ofensas ambientais; A seção 3 traz uma breve revisão da literatura empírica de crimes ambientais, inclusive para o Brasil; as seções 4 e 5 apresentam os dados, o modelo empírico e os resultados das estimações para a oferta de crimes ambientais nos estados brasileiros, considerando os agentes privados; e, por fim, a seção 6 traz as considerações finais do trabalho.

\section{Referencial Teórico ${ }^{2}$}

Becker (1968) assume que a atitude criminosa decorre de uma análise racional dos custos e dos benefícios envolvidos, comparados com o retorno em dedicar tempo e recursos em atividades legais. Ou seja, a decisão de cometer um crime ambiental resulta da maximização da utilidade esperada do agente, na qual o indivíduo pondera, por um lado, entre os potencias ganhos da ação criminosa e o valor da punição e as probabilidades de detenção, e, por outro lado, o custo de oportunidade de cometer crime. Então, por exemplo, uma firma, ao decidir se despeja os dejetos proibidos por lei em um rio, pondera os ganhos da infração com o valor da multa e a probabilidade de ser descoberta, além das demais sanções que sofrerá.

Segundo o modelo proposto por Becker, as ofensas cometidas contra o meio ambiente por parte de um agente racional dependem da probabilidade do fato acarretar em detenção, da magnitude da punição se condenado e de outras variáveis, como a renda disponível em atividades legais ou ilegais, o desejo de cometer crimes, etc.

Em termos formais, a oferta de crime ambiental em termos individuais é dada pela seguinte relação:

$$
A_{i}=A_{i}\left(c_{i}, p_{i}, d_{i}\right)
$$

em que $A_{i}$ são os autos de infração ambiental que um indivíduo $i$ pode cometer durante um determinado período, $c_{i}$ é a probabilidade de condenação por ofensa, $p_{i}$ é a punição por ofensa e $d_{i}$ contém as demais variáveis que influenciam a decisão do criminoso. As distribuições das variáveis $c_{i}$ e $p_{i}$ dependem do posicionamento das autoridades da jurisdição que enfrentar o infrator. A distribuição de $d_{i}$ depende das probabilidades de condenação e das punições de atividades ilegais concorrentes.

2 Nesta seção apresentamos o modelo de crime proposto em Becker (1968), considerando a questão ambiental. Esta seção está baseada em Uhr e Uhr (2014). 
Como somente os ofensores condenados são de fato punidos, existe "discriminação de preço" e incerteza: se condenado, paga $p_{i}$ pela ofensa, com $p_{i}>0$, caso contrário, não paga pelo crime $\left(p_{i}=0\right)$. Um aumento em $c_{i}$ ou em $p_{i}$ reduz a utilidade esperada de uma ofensa ambiental e, portanto, tende a reduzir o número de ofensas devido à probabilidade de "pagar um preço maior". Com relação aos efeitos de $d_{i}$, temos que um aumento na renda de atividades legais aumentaria o custo de oportunidade do crime, implicando um número de ofensas menor. Uma mudança na forma da punição, por exemplo, de uma multa para encarceramento, também tenderia a reduzir o número de infrações.

A oferta total de ofensas $(A)$ é a soma de todas as ofertas individuais $\left(A_{i}\right)$ e depende do conjunto de $c_{i}, d_{i}$ e $p_{i}$. Apesar de essas variáveis diferirem bastante entre os agentes, para simplificar, consideram os seus valores médios. Então, a função de mercado de ofensas ambientais pode ser escrita como:

$$
A=A(c, p, d)
$$

Neste trabalho, o objetivo é testar se os agentes privados afetam a oferta de crimes contra o meio ambiente através do aumento da probabilidade de condenação (c) e do valor da punição ( $p$ ). Ou seja, a ideia é que os agentes privados ajudam a monitorar e a delatar firmas infratoras, implicando aumento da probabilidade de condenação de um criminoso ambiental. Além disso, os agentes privados podem atuar diretamente sobre a variável de valor da punição ( $p$ ) quando divulgam para a sociedade civil informações negativas relacionadas às firmas poluidoras, implicando estigma moral e perdas de reputação (COHEN, 2000; ALMER; GOESCHL, 2010). Desse modo, espera-se um impacto negativo das ações privadas sobre a oferta de crimes ambientais no Brasil.

\section{Referencial Empírico 3}

A literatura empírica internacional que verifica os impactos de ações formais e informais sobre a oferta de crimes ambientais é ampla. Muitos dos trabalhos verificam os impactos dessas ações sobre as infrações através dos canais econômicos comentados no modelo teórico da seção anterior. Já a literatura empírica brasileira sobre o tema é escassa.

Podemos considerar dois grupos de sanções contra os agentes poluidores: as ações formais - dos órgãos governamentais - e as ações informais - de agentes privados. Pesquisas anteriores, como de Afsah et al. (1996), focaram a atenção sobre os efeitos das sanções formais sobre a inibição das ofensas ambientais. Em termos

3 Para um referencial mais amplo, ver Uhr e Uhr (2014). 
estritos, os artigos avaliam, por exemplo, o impacto de inspeções e da ameaça de inspeções para as taxas de adequação às normas de poluição da água e emissões de resíduos na indústria (MAGAT; VISCUSI, 1990; LAPLANTE; RILSTONE, 1996). Outros demonstram o impacto de sanções não monetárias para as taxas de cumprimento da legislação de poluição do ar (GRAY; DEILY, 1996).

A literatura atual tem avançado no sentido de verificar a importância do cumprimento das sanções. Almer e Goeschl (2010) estudam o efeito da execução criminal sobre ofensas ambientais na Alemanha. Os autores concluem que sanções criminais têm papel impeditivo relevante sobre os crimes ambientais. Shimshack e Ward (2008) argumentam que as sanções criminais não só têm um papel impeditivo importante, como também garantem o cumprimento da legislação acima do esperado. Ainda considerando as sanções, Shimshack e Ward (2005) estudam o impacto da multa marginal sobre a taxa de crimes ambientais cometidos, considerando a reputação do regulador e os possíveis efeitos de transbordamento espacial. Earnhart (2004) tem como objetivo determinar a efetividade das intervenções governamentais (inspeções e políticas de aplicação da lei a nível estadual e federal) no desempenho ambiental das firmas. Seus resultados mostram que existe um forte efeito impeditivo gerado pela ameaça de intervenção. Além disso, a real aplicação de sanções em nível estadual e federal também induz a um melhor desempenho ambiental das firmas. De igual forma, Stafford (2002) examina o impacto do aumento das penalidades para a disposição ilegal de resíduos perigosos. Ele conclui que os resultados são consistentes com o modelo de Becker (1968).

Além das ações formais, diversos trabalhos ressaltam a importância das sanções informais. Afsah et al. (1996) estudam se o papel do governo como regulador é complementado pela comunidade e o mercado, através do monitoramento. Ou seja, desejam responder se o desempenho ambiental dos agentes poluidores é influenciado pelos vizinhos de uma firma ou pelos consumidores dos produtos da firma poluidora. Segundo os autores, em comunidades mais ricas, bem-educadas e organizadas existem diversas formas de garantir o cumprimento das normas ambientais. Se a regulação formal está presente, as comunidades utilizam o processo político para influenciar o rigor no cumprimento da lei. Já se a ação do governo é ineficiente ou ausente, a "regulação informal" é implantada por grupos comunitários e ONGs ambientais. Os autores argumentam que, tanto para os países da OCDE quanto para países em desenvolvimento, os dados têm mostrado que a reputação ambiental das firmas importa e que os lucros e custos dessas firmas são afetados pelo julgamento de consumidores, acionistas e fornecedores. Segundo Afsah et al. (1996), "Em países tão distintos quanto China, Brasil, Indonésia e os Estados Unidos, muita da variação no desempenho ambiental das fábricas é expli- 
cado por variações inter-comunidades na renda, educação e poder de barganha" (AFSAH et al., 1996, p. 6).

Seguindo essa linha, Dasgupta, Hettige e Wheeler (2000) avaliam o impacto das características da firma, mercados, regulação formal e informal (pressão da comunidade) sobre o controle da poluição para fábricas mexicanas. Já Anton et al. (2004) estudam a influência de incentivos de mercado sobre a adoção voluntária de sistemas de gestão ambiental, como o padrão ISO 14000, e o efeito destes sobre a intensidade de emissões de poluentes tóxicos do ar por parte das empresas. Os resultados mostram que as pressões de consumidores e investidores, juntamente com a possibilidade de punição e a escala passada de emissões, são os determinantes mais importantes para a adoção de estratégias de gestão ambiental.

Em relação às evidências empíricas para o Brasil, três trabalhos merecem destaque. Ferraz e Seroa da Motta (2002) avaliam os impactos da regulação formal e informal sobre a decisão de investimento ambiental em indústrias do estado de São Paulo. Os autores sugerem que a organização local e a pressão da comunidade têm impacto indireto sobre a firma, através de reclamações aos órgãos reguladores, e que a regulação formal não tem efeitos diretos sobre a decisão de investimento da firma. Seroa da Motta (2006) analisa as variáveis que determinam o desempenho ambiental de empresas brasileiras, considerando tanto as características das firmas, quanto as demandas das comunidades e os incentivos de mercado. Os resultados mostram que os incentivos de mercado adicionais têm um papel crucial sobre a decisão da firma quanto ao nível de controle ambiental. $\mathrm{Ou}$ seja, a gestão ambiental das empresas é altamente afetada pelo nível das sanções e existe clara motivação para evitar tais punições. As demandas dos reguladores se constituem no principal determinante da adoção de práticas ambientais. Por fim, Uhr e Uhr (2014), utilizando dados de infrações ambientais contra a flora, para os estados brasileiros, testam a reputação do regulador no combate a violações ambientais. As variáveis empregadas para testar a reputação são os valores das multas lavradas no estado (efeito específico) e nos estados vizinhos (efeito spillover). Os autores encontram que uma multa aplicada em um estado sinaliza de forma crível a da autoridade ambiental para aplicar multas em outros estados, amplificando o impacto desse tipo de sanção no Brasil.

\section{Dados e Método}

O objetivo da análise empírica é testar se os agentes privados (ONGs ambientais e comunidades) impactam significativamente na oferta de crimes ambientais. A literatura argumenta que esses agentes privados atuam através do monitoramento, além de atuar diretamente sobre sanções (estigma moral e perdas de repu- 
tação). Para a investigação econômica do crime ambiental, seguindo o argumento teórico de Becker (1968), procura-se estimar a seguinte equação:

$$
y_{i t}=\alpha+x_{i t} \beta+c_{i}+\varepsilon_{i t}
$$

em que $y_{\text {it }}$ é o logaritmo natural da oferta de crimes ambientais para o estado $i(i=1,2, \ldots, 27)$ no ano $t(t=2006,2007)$. O termo $\alpha$ representa a constante. $O$ vetor $\mathrm{x}_{\mathrm{it}}$ representa as variáveis explicativas, todas em logaritmo natural, e $\beta$ representa os parâmetros a serem estimados. $\mathrm{O}$ termo $c_{i}^{7}$ representa a heterogeneidade estadual, ou seja, contém as características específicas do estado i que não mudam no tempo. Por fim, $\varepsilon_{\mathrm{it}}$ é o termo do erro.

Dada as dimensões temporal e espacial da nossa amostra, a análise com técnicas de dados em painel é indicada. Como os dados em painel se relacionam a indivíduos, empresas, estados, etc., tende a haver muita heterogeneidade, nessas unidades, que não é observada explicitamente. Uma variável não observada e constante no tempo é chamada de efeito não observado ou heterogeneidade individual (o termo $c_{i}$ da equação de regressão). Essa variável captura características dos estados que não mudam no tempo, como, por exemplo, características culturais da população ou características geográficas. A motivação primária para usar dados de painel é resolver o problema da heterogeneidade individual, que é tratada como uma variável aleatória, e não um parâmetro a ser estimado. Além disso, possibilita o controle parcial do problema de erro de medida decorrente do sub-registro dos crimes ambientais.

Para medir a incidência de crimes ambientais, utiliza-se como variável dependente ${ }^{4}$ o número de autos de infração de poluição registrados pelo Instituto Brasileiro do Meio Ambiente e dos Recursos Naturais Renováveis (Ibama) nos estados brasileiros para os anos de 2006 e 2007. São registrados, em média, 159 autos de infração, do tipo poluição, por estado e por ano. Tais dados estão descritos na Tabela 1.

4 Aplicamos o logaritmo natural à normalização dos dados, ou seja, $\ln ([(x-\operatorname{Min}(\mathbf{x})) /(\operatorname{Max}(\mathbf{x})$ $\operatorname{Min}(\mathbf{x}))])$, em que x é o vetor dos autos de infração de poluição. 
Tabela 1 - Autos de infração de poluição registrados pelo Ibama

\begin{tabular}{|c|c|c|c|}
\hline Estado/Região & Ano 2006 & Ano 2007 & Média Anual \\
\hline $\mathrm{AC}$ & 4 & 2 & 3 \\
\hline $\mathrm{AM}$ & 50 & 32 & 41 \\
\hline $\mathrm{AP}$ & 31 & 14 & 22,5 \\
\hline PA & 174 & 204 & 189 \\
\hline $\mathrm{RO}$ & 105 & 272 & 188,5 \\
\hline $\mathrm{RR}$ & 18 & 14 & 16 \\
\hline TO & 48 & 306 & 42 \\
\hline Norte & 430 & 844 & 637 \\
\hline $\mathrm{AL}$ & 51 & 476 & 263,5 \\
\hline $\mathrm{BA}$ & 134 & 81 & 107,5 \\
\hline $\mathrm{CE}$ & 283 & 254 & 268,5 \\
\hline MA & 128 & 182 & 155 \\
\hline $\mathrm{PB}$ & 131 & 227 & 179 \\
\hline $\mathrm{PE}$ & 40 & 54 & 47 \\
\hline PI & 21 & 23 & 22 \\
\hline $\mathrm{RN}$ & 35 & 80 & 57,5 \\
\hline SE & 8 & 11 & 9,5 \\
\hline Nordeste & 831 & 1388 & 1109,5 \\
\hline $\mathrm{DF}$ & 146 & 158 & 152 \\
\hline GO & 356 & 235 & 295,5 \\
\hline MS & 315 & 220 & 267,5 \\
\hline MT & 251 & 376 & 313,5 \\
\hline Centro Oeste & 1068 & 989 & 1028,5 \\
\hline ES & 359 & 310 & 334,5 \\
\hline MG & 335 & 349 & 342 \\
\hline $\mathrm{RJ}$ & 409 & 217 & 313 \\
\hline SP & 105 & 74 & 89,5 \\
\hline Sudeste & 1208 & 950 & 1079 \\
\hline
\end{tabular}


conclusão.

\begin{tabular}{cccc}
\hline Estado/Região & Ano 2006 & Ano 2007 & Média Anual \\
\hline PR & 79 & 86 & 82,5 \\
RS & 136 & 230 & 183 \\
SC & 236 & 97 & 166,5 \\
Sul & $\mathbf{4 5 1}$ & $\mathbf{4 1 3}$ & $\mathbf{4 3 2}$ \\
\hline BRASIL & $\mathbf{3 9 9 8}$ & $\mathbf{4 5 8 4}$ & $\mathbf{4 2 9 1}$ \\
\hline
\end{tabular}

Fonte: Elaboração própria.

A Tabela 2 apresenta as variáveis explicativas a serem utilizadas no modelo empírico, indicando uma breve descrição e o sinal esperado dos coeficientes a serem estimados.

Tabela 2 - Variáveis explicativas para a incidência de crimes ambientais

\begin{tabular}{lll}
\hline Variável explicativa & Descrição & Sinal esperado \\
\hline Comunidade & $\begin{array}{l}\text { Taxa de urbanização: razão entre } \\
\text { população urbana e o total da } \\
\text { população do estado. }\end{array}$ & Negativo \\
ONGs & $\begin{array}{l}\text { Número de ONGs ambientais com } \\
\text { sede em cada estado. }\end{array}$ & Negativo \\
Rendapc & $\begin{array}{l}\text { PIB per capita (em milhares de } \\
\text { reais). }\end{array}$ & Positivo \\
Rendapc quadrado & $\begin{array}{l}\text { PIB per capita ao quadrado (em } \\
\text { milhares de reais). }\end{array}$ & Negativo \\
\hline
\end{tabular}

Fonte: Elaboração própria.

Nota: Para a análise empírica, todas as variáveis são utilizadas em logaritmo natural.

A variável "comunidade" caracteriza a concentração populacional do estado em centros urbanos, ou seja, é formada pela razão entre população urbana e o total da população estadual (IBGE, 2006, 2007). A taxa de urbanização serve como proxy para o papel das comunidades de monitorar e delatar agentes que comentem crimes contra o meio ambiente. Ou seja, a hipótese é que, quanto maior a taxa de urbanização em determinada localidade, maior é a facilidade de monitorar o comportamento da firma poluidora e informar as suas ações para os órgãos responsáveis. Uma vez que os agentes poluidores (como, por exemplo, as firmas poluidoras) estão fixos e seus atos de poluição são visíveis para a sociedade, não é plausível supor que consigam esconder a atividade criminosa por muito tempo. Assim, essa variável é utilizada para explicar o crime sob a justificativa de que, em estados com maior taxa de urbanização, existe maior troca de informações en- 
tre os indivíduos da sociedade, implicando maiores custos de execução do crime ambiental.

A variável "ONGs" é dada pelo número de ONGs ambientais com sede em cada estado cadastradas junto ao Ministério do Meio Ambiente e ao Conselho Nacional do Meio Ambiente (Conama). As ONGs ambientais também servem como proxy para o comportamento dos agentes privados, atuando como reveladoras de informação. Por um lado, relatam violações dos padrões ambientais às agências reguladoras, pressionando para a aplicação de sanções. Por outro lado, revelam o comportamento dos agentes poluidores para a sociedade através da mídia (UHR; UHR; MUELLER, 2012). Tal ação gera sanções morais e perdas de reputação para os agentes poluidores.

Quanto ao uso das variáveis "renda pc" e "renda pc quadrado" (IBGE, 2006, 2007), elas cumprem duas funções. Em primeiro lugar, aumentos de renda refletem aumentos na produção, indicando maior incidência de poluição e, provavelmente, de crimes contra o meio ambiente. Entretanto, conforme a sociedade enriquece, ocorre substituição da produção, de indústrias sujas para limpas, assim como avanços tecnológicos redutores da poluição, diminuindo a incidência de crimes ambientais. ${ }^{5}$ Em segundo lugar, a variável renda pode ser utilizada como proxy para o retorno da atividade ilegal. A ideia é que um aumento na renda do crime implica maior probabilidade do agente engajar-se na atividade criminal (EHRLICH, 1973), que pode apresentar retornos decrescentes de escala. A Tabela 3 apresenta as estatísticas descritivas.

Tabela 3 - Estatísticas descritivas para variáveis explicativas

\begin{tabular}{lll}
\hline Variável & Média & Desvio padrão \\
\hline Comunidade & 2,1542 & 0,2526 \\
ONGs & 2,2944 & 1,2017 \\
Rendapc & 1,7617 & 0,5023 \\
\hline
\end{tabular}

Fonte: Elaboração própria.

Nota: Média e desvio padrão calculados a partir dos logaritmos naturais das variáveis.

A Tabela 3 complementa as informações apresentadas na tabela anterior, isto é, descreve as médias e os desvios padrão das variáveis explicativas utilizadas no modelo empírico. Cabe destacar que são os valores médios das variáveis em logaritmo natural.

5 Conforme expresso pela literatura da curva de Kuznets ambiental (GROSSMAN; KRUEGER, 1995). 


\section{Resultados}

A Tabela 4 apresenta as estimativas dos coeficientes para o modelo de crimes ambientais proposto anteriormente. Na primeira estimativa (coluna 1), utiliza-se o modelo de mínimos quadrados ordinários (MQO) com dados empilhados, em que o efeito individual de cada estado não é levado em consideração. As demais colunas apresentam os resultados estimados por dados em painel. Na coluna 2 temos a regressão por efeitos aleatórios, e a coluna 3 apresenta a regressão por efeitos fixos.

Tabela 4 - Modelo econométrico para crimes ambientais

\begin{tabular}{llll}
\hline $\begin{array}{l}\text { Variável } \\
\text { independente }\end{array}$ & $\begin{array}{l}\text { MQO empilhados } \\
(\mathbf{1})\end{array}$ & $\begin{array}{l}\text { Efeito aleatório } \\
(\mathbf{2})\end{array}$ & Efeito fixo (3) \\
\hline Rendapc & 1,0689 & 2,8986 & $16,3659^{* * *}$ \\
& $(0,475)$ & $(0,173)$ & $(0,006)$ \\
Rendapc quad- & $-0,2878$ & $-0,7199$ & $-3,5608^{* *}$ \\
rado & $(0,459)$ & $(0,195)$ & $(0,027)$ \\
& $0,5913^{* * *}$ & $0,5144^{* * *}$ & $-2,8276^{* *}$ \\
ONGs & $(0,000)$ & $(0,006)$ & $(0,043)$ \\
& $-0,4001$ & $-0,8887^{*}$ & $-1,2068^{* *}$ \\
Comunidade & $(0,469)$ & $(0,074)$ & $(0,032)$ \\
& $-2,8988$ & $-3,4376$ & $-9,1450$ \\
Constante & $(0,123)$ & $(0,129)$ & $(0,112)$ \\
& 0,3942 & 0,3633 & 0,2436 \\
R2 & - & 18,34 & - \\
Hausman test & & $(0,0011)$ & \\
& - & 11,35 & - \\
Breusch-Pagan test & & $(0,0008)$ & \\
\hline Observações & 54 & 54 & 54 \\
\hline
\end{tabular}

Fonte: Elaboração própria.

Nota: Os valores entre parênteses são o p-value das variáveis. O nível de significância estatística é dado por: $*=10 \% ;{ }^{* *}=5 \% ; * *=1 \%$; todas as variáveis encontram-se em logaritmo natural. 
Ao aplicar o teste de Breusch-Pagan ao modelo de efeito aleatório, o qual testa a existência de efeitos individuais não observáveis, conclui-se que existe componente de heterogeneidade a 1\% de confiança. Ou seja, rejeita-se a hipótese nula de não existência de efeito aleatório não observável. Logo, a estimação por MQO empilhados é viesada e inconsistente. Dada a presença de heterogeneidade, aplicou-se o teste de Hausman para verificar se o componente não observado é correlacionado com as variáveis explicativas. O resultado do teste de Hausman indicou correlação a 1\% de confiança, revelando que a estimação por efeitos aleatórios não gera estimadores consistentes para o modelo. Logo, a estimativa por efeitos fixos é preferível.

Os sinais obtidos para as variáveis "renda pc" e "renda pc quadrado" se mantiveram para os três modelos e confirmam as hipóteses anteriores. Analisando o modelo de efeitos fixos, a renda per capita apresenta impacto positivo sobre a taxa de crimes ambientais com um nível de significância de 1\%, e o efeito marginal decrescente também é significativo a 5\% de confiança.

Quanto à variável "ONGs", apesar de apresentar resultado significativo a $1 \%$ de confiança nas estimações por MQO empilhados e efeitos aleatórios, os sinais dos coeficientes foram contrários ao esperado. Como dito anteriormente, não podemos considerar esses resultados devido à existência do componente não observado e sua correlação com as variáveis explicativas. Assim, pelo modelo de efeitos fixos, percebe-se que a variável "ONGs" apresenta impacto negativo e significativo a $5 \%$ de confiança, sustentando a hipótese de que a presença das ONGs ajuda a reduzir a ocorrência de crimes ambientais, tanto como reveladoras de informação, monitorando e delatando o comportamento das firmas criminosas, como atuando de forma direta, através de sanções morais que geram perdas de reputação para os agentes poluidores (COHEN, 2000; ALMER; GOESCHL, 2010; UHR; UHR; MUELLER, 2012).

A outra variável utilizada para identificar os agentes privados foi a atuação das comunidades. Os resultados mostram sinal negativo em todas as estimações. Na estimação por efeitos fixos a variável "comunidade" é significante a 5\% de confiança. Esse resultado sustenta a hipótese de que estados com maior taxa de urbanização apresentam maior troca de informações entre os indivíduos sobre as atividades criminosas dos agentes poluidores, impondo custos adicionais para os infratores.

Desse modo, aceitou-se a hipótese de que os agentes privados influenciam negativamente a oferta de crimes ambientais no Brasil. Ou seja, quanto mais forte a fiscalização por parte das comunidades e das ONGs ambientais, maior é o custo para o agente criminoso. O aumento do custo do crime implica redução na oferta de crimes contra o meio ambiente. Esse resultado sugere que as estimações que 
buscam determinar os componentes socioeconômicos dos crimes ambientais devem considerar os agentes privados como forma de controle.

\section{Considerações Finais}

O objetivo deste trabalho consistiu em apresentar evidências para o papel dos agentes privados no combate a crimes ambientais. Conforme visto, a oferta de crimes no modelo de Becker (1968) é determinada pela probabilidade de condenação, pela intensidade da punição aplicada e por outras variáveis, tais como renda disponível em atividades legais ou ilegais e o desejo de cometer crimes. A probabilidade de condenação é tanto maior quanto maiores forem os gastos do governo e os gastos privados com monitoramento. Desse modo, os agentes privados influenciam a oferta de crimes ambientais diretamente através do monitoramento. Além disso, a literatura recente argumenta que os agentes privados podem aplicar "sanções" extralegais sobre os criminosos, tais como o estigma moral e perdas de reputação (COHEN, 2000).

$\mathrm{Na}$ análise empírica, testaram-se os efeitos dos agentes privados sobre as taxas de crime ambiental (autos de infração de poluição) no Brasil. A análise considerou dados em painel para todos os estados brasileiros para os anos de 2006 e 2007, obtidos junto ao Instituto Brasileiro do Meio Ambiente e dos Recursos Naturais Renováveis (Ibama). Utilizamos as técnicas de MQO empilhados e dados em painel por efeitos aleatórios e efeitos fixos. Como variáveis para os agentes privados foram utilizadas o número de ONGs ambientais e a taxa de urbanização estadual (comunidade). A conclusão empírica é que as ONGs ambientais e as comunidades de fato ajudam a reduzir a oferta de crimes ambientais.

Cabe destacar que apesar da dificuldade de encontrar dados sobre o meio ambiente no Brasil, e da limitação às análises mais complexas que isso causa, este trabalho inova na medida em que proporciona evidências da importância dos agentes privados no combate ao crime ambiental no Brasil. Sugerimos às pesquisas futuras o aprimoramento da base de dados e/ou o estudo dos agentes privados em nível municipal, ou, até mesmo, o estudo de como comunidades específicas agiram no combate ao crime ambiental.

As evidências encontradas neste trabalho indicam que estimações que buscam determinar os componentes socioeconômicos dos crimes ambientais devem considerar os agentes privados como forma de controle. Além disso, pelos resultados pode-se argumentar que políticas públicas direcionadas aos agentes privados imporiam custos adicionais sobre os agentes criminosos. 


\section{Referências}

AFSAH, S.; LAPLANTE, B.; WHEELER, D. Controlling industrial pollution: a new paradigm. Washington, D.C: The World Bank, Oct. 1996. (Policy research Working Paper, n. 1672).

ALMER, C.; GOESCHL, T. Environmental crime and punishment: empirical evidence from the German penal code. Land Economics, v. 86 n. 4, p. 707-726, 2010.

ANTON, W. R. Q.; DELTAS, G.; KHANNA, M. Incentives for environmental self-regulation and implications for environmental performance. Journal of Environmental Economics and Management, v. 48, n. 1 p. 632-654, July 2004.

BECKER, G. S. Crime and punishment: an economic approach. The Journal of Political Economy, v. 76, n. 2, p. 169-217, 1968.

BRASIL. Ministério do Meio Ambiente. Conselho Nacional do Meio Ambiente. Disponível em: <http://www.mma.gov.br/port/conama >. Acesso em: 12 fev. 2011.

COHEN, M. A. Empirical research on the deterrent effect of environmental monitoring and enforcement. The Environmental Law Reporter, v. 30, p. 10245-10252, Apr. 2000.

DASGUPTA, S.; HETTIGE, H.; WHEELER, D. What improves environmental compliance? Evidence from Mexican industry. Journal of Environmental Economics and Management, v. 39, n. 1, p. 39-66, Jan. 2000.

EARNHART, D. Regulatory factors shaping environmental performance at publicly-owned treatment plants. Journal of Environmental Economics and Management, v. 48, n. 1, p. 655681, July 2004.

EHRLICH, I. Participation in illegitimate activities: a theoretical and empirical investigation. Journal of Political Economy, v. 81, n. 3, p. 526-536, May-June 1973.

FERRAZ, C.; SEROA DA MOTTA, R. Regulação, mercado ou pressão social? Os determinantes do investimento ambiental na indústria. Rio de Janeiro: IPEA, mar. 2002. (Texto para Discussão, n. 863).

GRAY, W.; DEILY, M. Compliance and enforcement: air pollution regulation in the U.S. steel industry. Journal of Environmental Economics and Management, v. 31, n. 1, p. 96-111, July 1996.

GROSSMAN, G. M.; KRUEGER, A. B. Economic growth and the environment. Quarterly Journal of Economics, v. 110, n. 2, p. 353-377, 1995.

HELLAND, E. The enforcement of pollution control laws: inspections, violations, and selfreporting. Review of Economics and Statistics, v. 80, n. 1, p. 141-153, Mar. 1998.

IBGE. Pesquisa Nacional por Amostra de Domicílios. Síntese de Indicadores 2006. 2006. Disponível em: <http:/www.ibge.gov.br/home/estatistica/populacao/trabalhoerendimento/ pnad2006/default.shtm>. Acesso em: 10 jan. 2011. 
IBGE. Pesquisa Nacional por Amostra de Domicílios. Síntese de Indicadores 2007. Disponível em: <http://www.ibge.gov.br/home/estatistica/populacao/trabalhoerendimento/pnad2007/ default.shtm>. Acesso em: 10 jan. 2011.

INSTITUTO BRASILEIRO DO MEIO AMBIENTE E DOS RECURSOS NATURAIS RENOVÁVEIS. Sobre a Lei de Acesso a Informação: acesso à informação. Disponível em: <http://www.ibama. gov.br/acesso-a-informacao/acesso-a-informacao>. Acesso em: 13 maio 2011.

LAPLANTE, B.; RILSTONE, P. Environmental inspections and emissions in the pulp and paper industry in Quebec. Journal of Environmental Economics and Management, v. 31, n. 1, p. 19-36, July 1996.

MAGAT, W. A.; VISCUSI, W. K. Effectiveness of the EPA's regulatory enforcements: the case of industrial effluent standards. Journal of Law and Economics, v. 30, n. 2, p. 331-360, Oct. 1990.

NADEAU, L. W. EPA effectiveness at reducing the duration of plant-level noncompliance. Journal of Environmental Economics and Management, v. 34, n. 1, p. 54-78, Sept. 1997.

SEROA DA MOTTA, R. Analyzing the environmental performance of the Brazilian industrial sector. Ecological Economics, v. 57, n. 2, p. 269-281, May 2006.

SHIMSHACK, J. P.; WARD, M. B. Enforcement and over-compliance. Journal of Environmental Economics and Management, v. 55, n. 1, p. 90-105, Jan. 2008.

. Regulator reputation, enforcement, and environmental compliance. Journal of Environmental Economics and Management, v. 50, n. 3, p. 519-540, Nov. 2005.

STAFFORD, S. L. The effect of punishment on firm compliance with hazardous waste regulation. Journal of Environmental Economics and Management, v. 44, n. 2, p. 290-308, Sept. 2002.

UHR, D. A. P.; UHR, J. G. Z.; MUELLER, B. Como as ONGs ambientais influenciam a política ambiental brasileira? Revista Brasileira de Economia, v. 66, n. 1, p. 79- 98, jan.-mar. 2012.

UHR, J. G. Z.; UHR, D. A. P. Infrações Ambientais e a Reputação do Regulador: Análise em Dados de Painel para o Brasil. Estudos Econômicos, São Paulo, v. 44, n. 1, p. 69-103, 2014.

Recebido em: 12/08/11. Aceito em: 25/03/14. 